\title{
Polymer ligand design and surface modification of Ag nanowires toward color-tone-tunable transparent conductive films
}

Shoma Kitamura, ${ }^{\dagger}$ Motoyuki Iijima, ${ }^{\ddagger},{ }^{*}$ Junichi Tatami, ${ }^{\ddagger}$ Tsubasa Fuke,,${ }^{\zeta}$ Takashi Hinotsu, ${ }^{\zeta}$ Kimitaka Sato $^{5}$

${ }^{\dagger}$ Graduate School of Engineering Science, Yokohama National University, 79-5 Tokiwadai, Hodogayaku, Yokohama, Kanagawa 240-8501, Japan

\#Faculty of Environment and Information Sciences, Yokohama National University, 79-7 Tokiwadai,

Hodogayaku, Yokohama, Kanagawa 240-8501, Japan

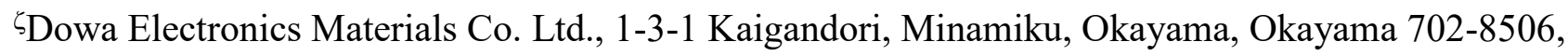
Japan

*Corresponding author. E-mail address: iijima@ynu.ac.jp (M. Iijima); Phone \& Fax: +81-45-339-3958. 
Calculation of adsorbed polymer content on Ag nanowires from TG curves. The polymer contents

on Ag nanowires were calculated from the following equation;

$$
\left(W_{700}-W_{200}\right) /\left(W_{\text {sample }}-W_{700}\right) \times 100
$$

where $W_{\text {sample }}[\mathrm{g}], W_{200}[\mathrm{~g}]$, and $W_{700}$ [g] are the initial sample weight, weight loss at $200^{\circ} \mathrm{C}$, and weight loss at $700^{\circ} \mathrm{C}$, respectively, during the TGA analysis. 


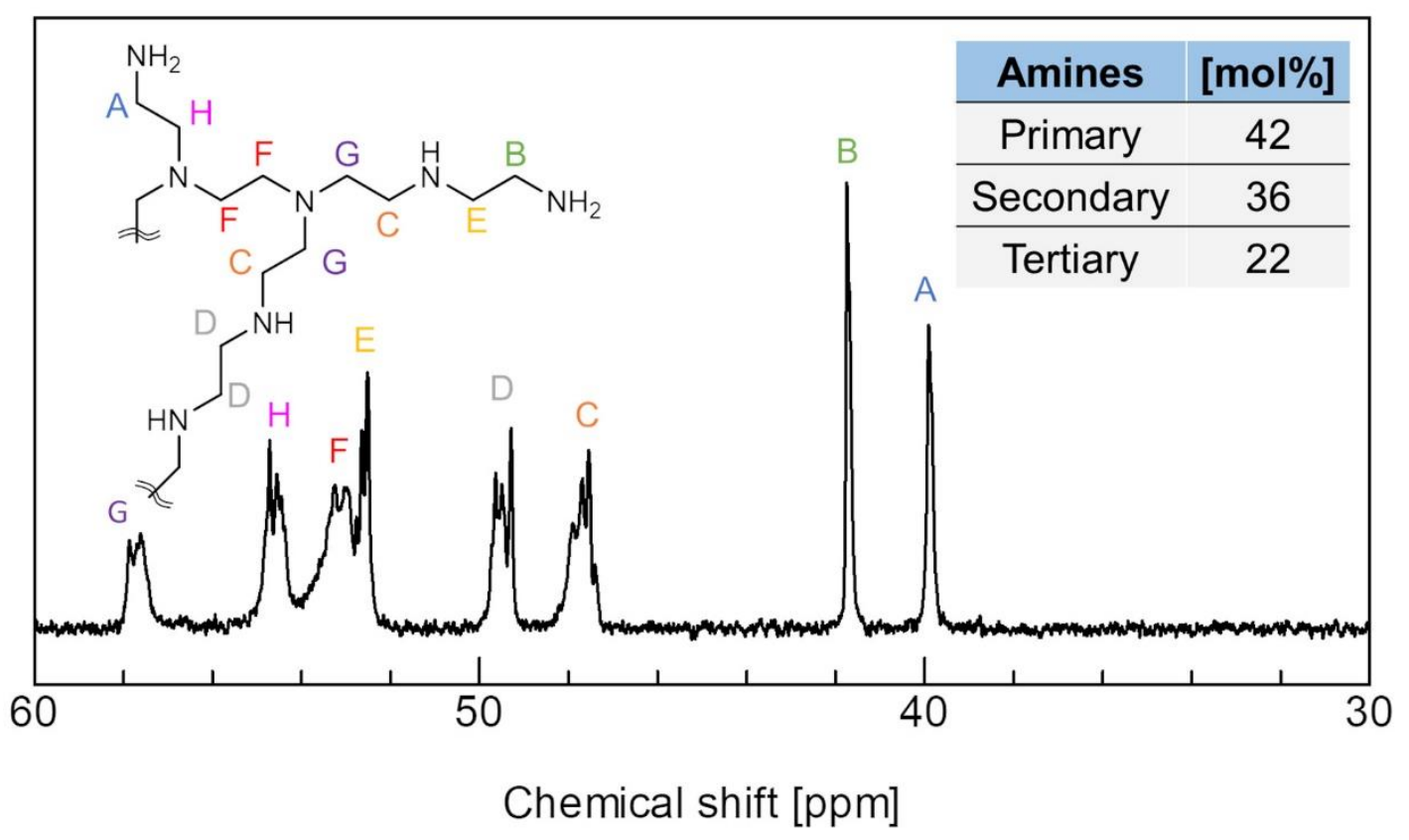

Figure S1 13C NMR of PEI. The ratio of primary, secondary, and tertiary amine was evaluated based on previous report [ref 35 in the manuscript]. 

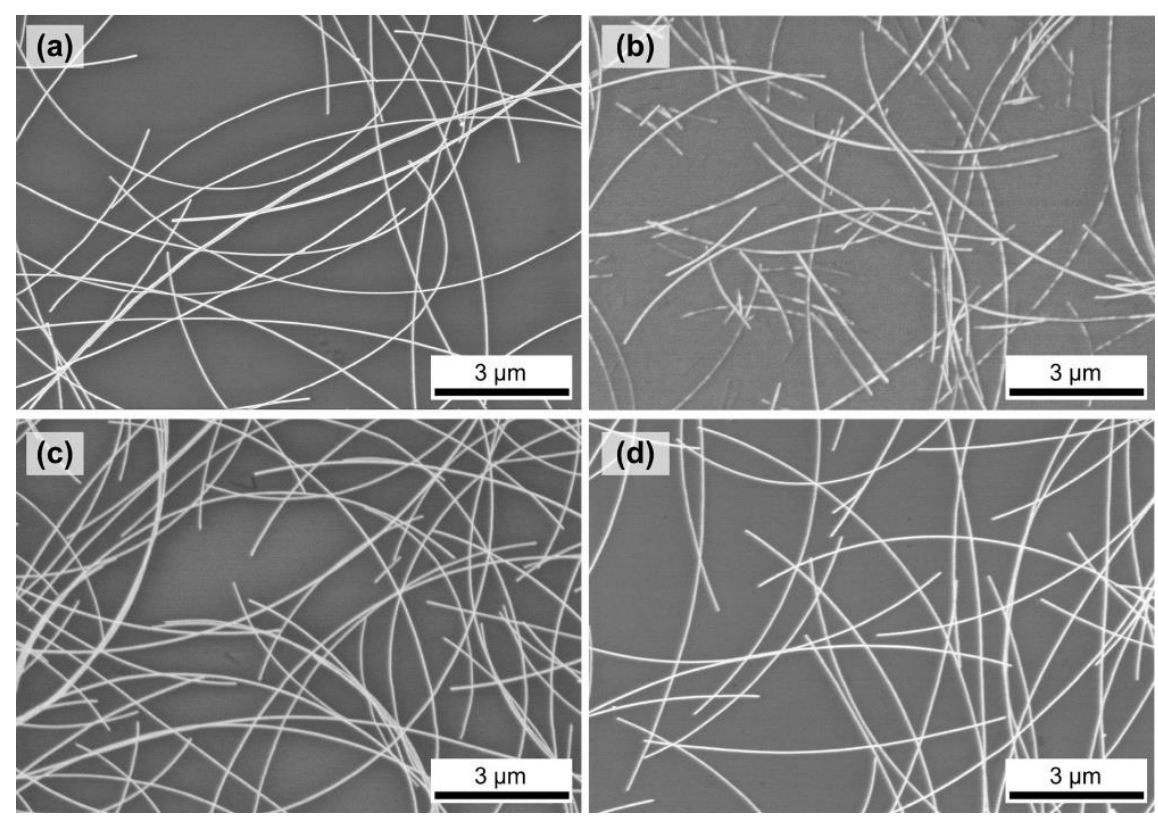

Figure S2 FE-SEM images of Ag nanowires casted on PET film; Ag nanowires (a) before (rod No. 3)

and after ligand exchange process using (b) PEI (rod No. 10), (c) PEI-mPEG20 (rod No. 8), and (d) PEImPEG40 (rod No. 4). 

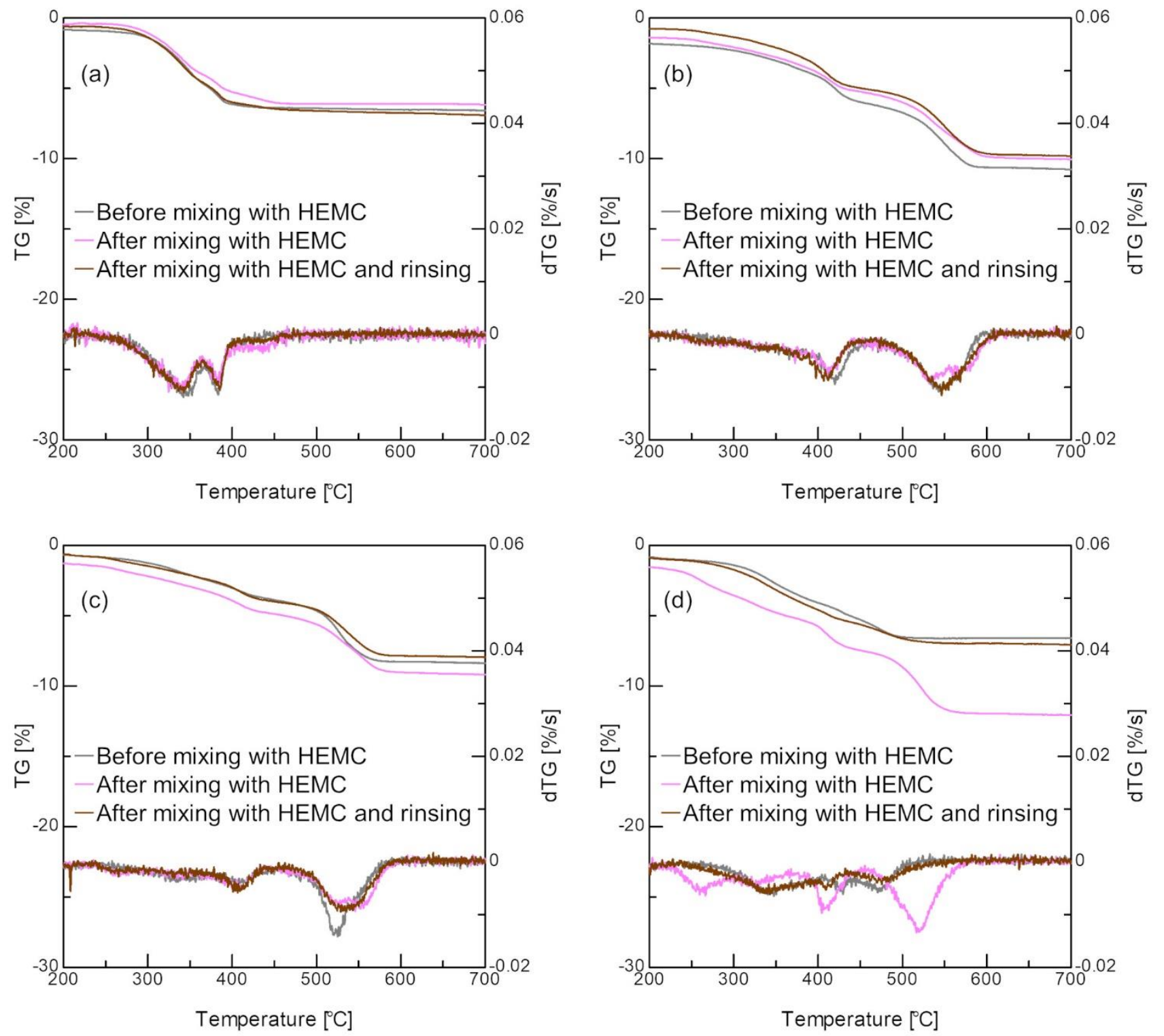

Figure S3 TGA and dTG profiles of Ag nanowires (a) before and after ligand exchange process using

(b) PEI, (c) PEI-mPEG20, and (d) PEI-mPEG40. 

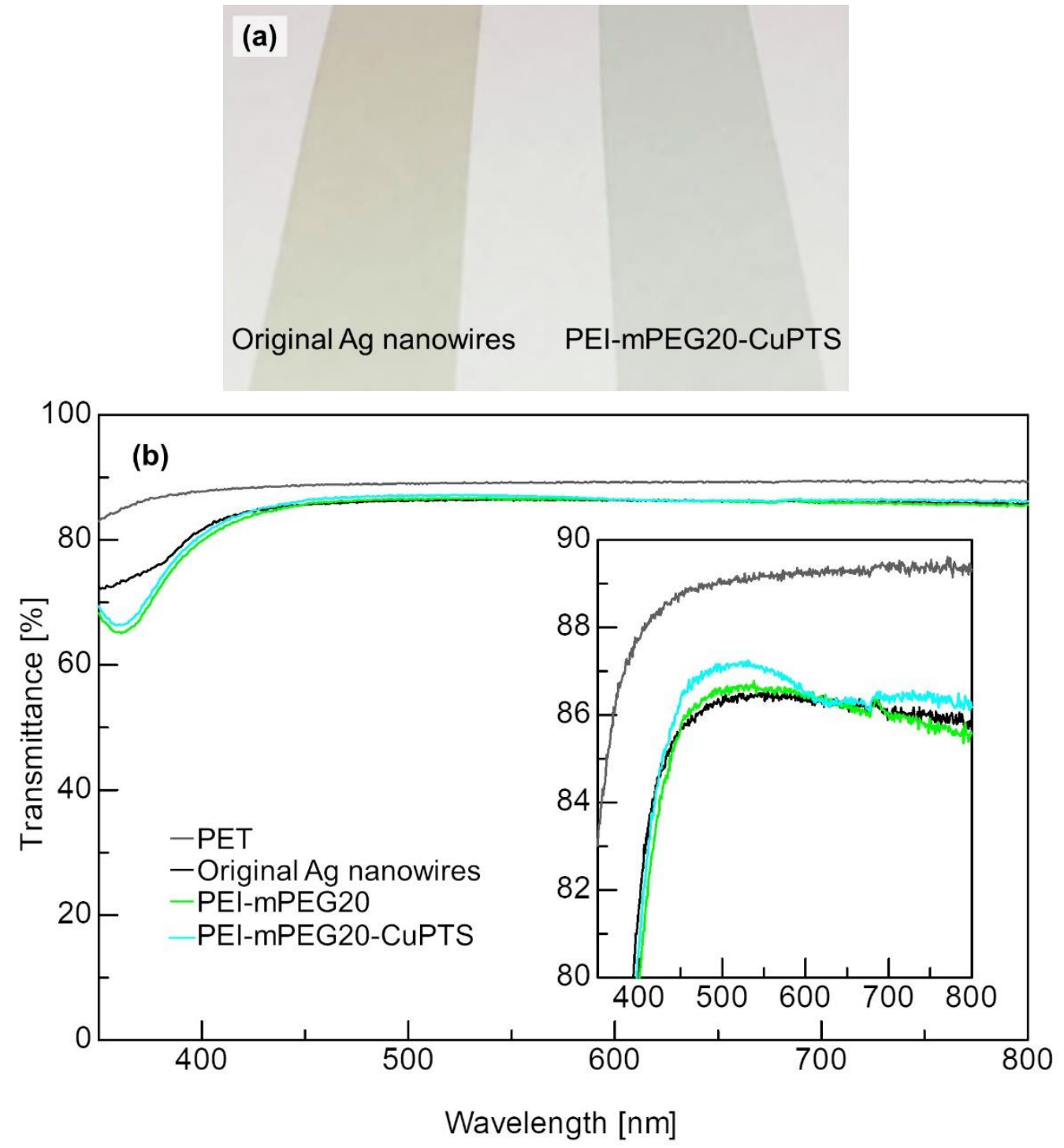

Figure S4 (a) Visible image of Ag nanowire film prepared by using original Ag nanowires (rod No. 14) and Ag nanowires loaded with CuPTS on PEI-mPEG20 (rod No. 14). (b) Transmittance of PET films and Ag nanowire films prepared by using original Ag nanowires (rod No. 7), Ag nanowires ligand exchanged to PEI-mPEG20 (rod No. 14), and Ag nanowires further loaded with CuPTS (rod No. 14). 


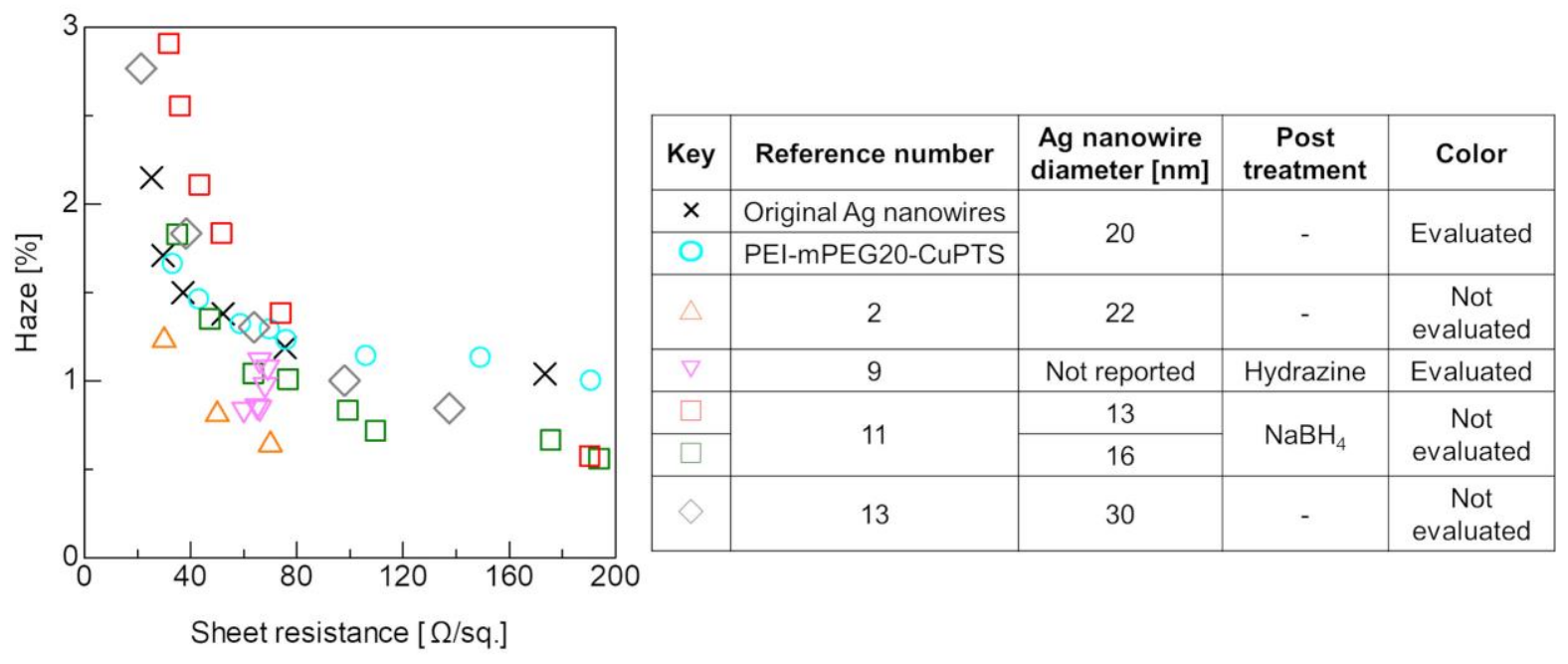

Figure S5 Haze and sheet resistance values of Ag nanowire films compared with those reported in other studies (data adopted from ref. 2, 9, 11, and 13). 


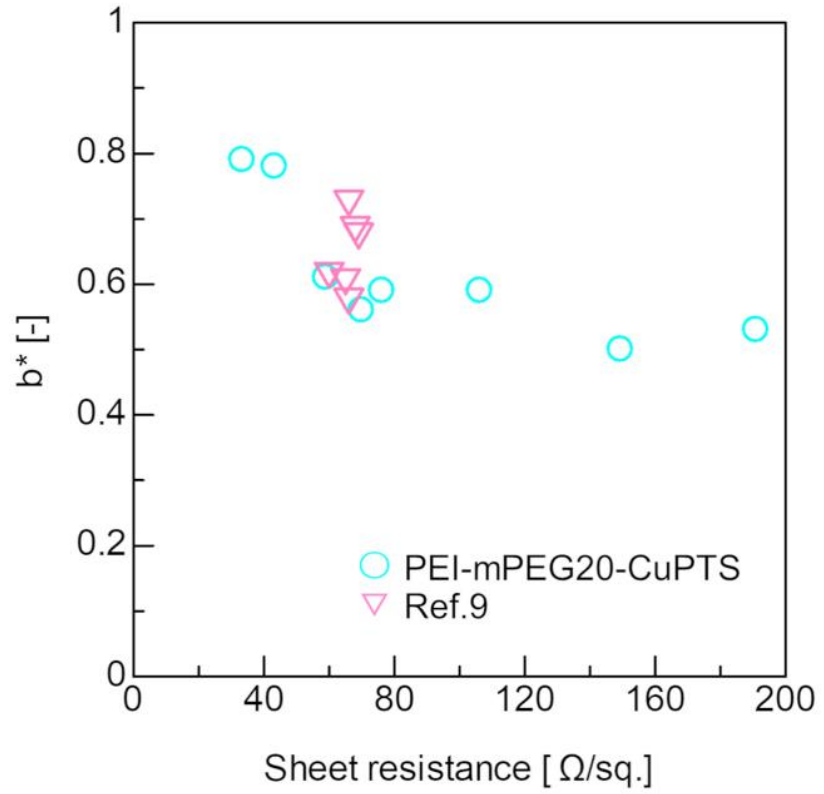

Figure S6 b* values of Ag nanowire films compared with those reported in other study (data adopted from ref. 9). 


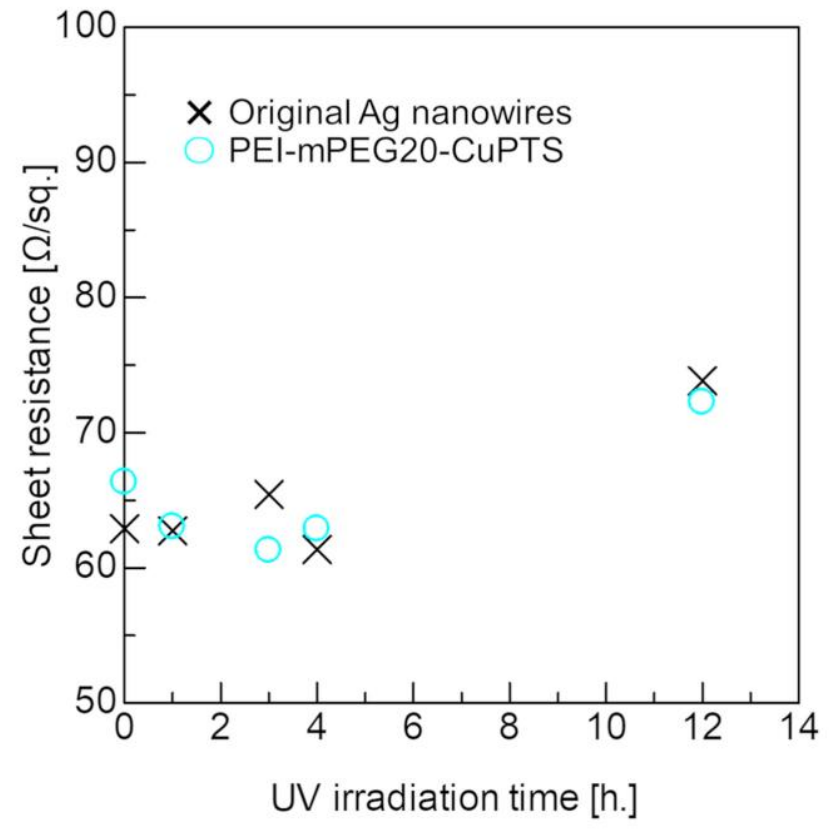

Figure S7 Accelerated degradation tests of Ag nanowire films. The changes in sheet resistance of Ag nanowire films which were settled under UV light $\left(4 \mathrm{~mW} / \mathrm{cm}^{2}\right.$ at $\left.365 \mathrm{~nm}\right)$ were monitored. 